\title{
MILLOUX INEQUALITY OF NONLINEAR DIFFERENCE MONOMIALS AND ITS APPLICATION
}

\author{
ZhaOJUn Wu AND Hongyan Xu
}

Abstract. Let $f(z)$ be a transcendental meromorphic function of finite order and $c_{1}, c_{2}, \cdots, c_{m}$ be complex constants satisfying that at least one of them is non-zero. The authors establish an inequality (Milloux inequality) about the nonlinear difference monomials $f^{d_{1}}\left(z+c_{1}\right) f^{d_{2}}(z+$ $\left.c_{2}\right) \cdots f^{d_{m}}\left(z+c_{m}\right)$, where $d_{1}, d_{2}, \cdots, d_{m} \in \mathbb{N}$. As an application of the inequality, the authors investigate the value distribution of $f^{d_{1}}\left(z+c_{1}\right) f^{d_{2}}\left(z+c_{2}\right) \cdots f^{d_{m}}\left(z+c_{m}\right)$. Results obtained partially promote and improve relevant results of Laine, Yang and Chen et al..

Mathematics subject classification (2010): 30D30.

Keywords and phrases: Milloux inequality, nonlinear, difference monomials, meromorphic function.

\section{REFERENCES}

[1] Z. X. CHEN, On growth, zeros and poles of meromorphic Solutions of linear and nonlinear difference equations, Sci China Math, 54 (2011), 2123-2133.

[2] Z. X. Chen, Z. B. Huang And X. M. Zheng, On properties of diference polynomials, Acta Math. Sci., 31B (2011), 627-633.

[3] Y. M. ChiAng And S. J. Feng, On the Nevanlinna characteristic of $f(z+\eta)$ and difference equations in the complex plane, Ramanujan J., 16 (2008) 105-129.

[4] Y. M. ChiAng AND S. J. Feng, On the growth of logarithmic difference, difference equations and logarithmic derivatives of meromorphic functions, J. Trans. Amer. Math. Soc., 361 (2009) 3767-3791.

[5] G. Gundersen, Finite order solutions of second order linear differential equations, Trans Amer Math Soc, 305 (1988), 415C429.

[6] R. G. Halburd And R. J. Korhonen, Nevanlinna theory for the difference operator, Ann. Acad. Sci. Fenn. Math., 31 (2006) 463-478.

[7] R. G. HalbuRd AND R. J. KorHONEn, Meromorphic solutions of difference equations, integrability and the discrete Painleve equations, J. Phys. A: Math. Theor. 40 (2007), 1-38.

[8] R. G. Halburd AND R. J. Korhonen, Difference analogue of the lemma on the logarithmic derivative with applications to difference equations, J. Math. Anal. Appl. 314 (2006), 477-487.

[9] W. K. HaYman, Meromorphic functions, Oxford Mathematical Monographs Clarendon Press, Oxford 1964.

[10] I. LAINE AND C. C. YANG, Value distribution of difference polynomials, Proc. Japan Acad. Ser A., 83 (2007), 148-151.

[11] C. C. YANG AND H. X. YI, Uniqueness theory of meromoprhic functions, vol. 557 of Mathematics and Its Application, Kluwer Academic Publishers, Dordrecht, The Netherlands, 2003.

[12] L. YANG, Value distribution theory, Translated and revised from the 1982 Chinese original. SpringerVerlag, Berlin; Science Press Beijing, Beijing, 1993.

[13] H. X. YI, Value distribution of $f^{\prime} f$, Chinese Science Bulletin, 34, 10 (1989), 727-730.

[14] R. R. ZHENG AND Z. X. CHEN, Value distribution of difference polynomials of meromorphic functions (in Chinese), Sci. Sin. Math. 42, 11 (2012), 1115-1130.

[15] R. R. ZHENG AND Z. X. CHEN, Fixed points of meromorphic functions and of their difference, divided differences and shifts, Acta Mathematics Sinica, English Series, 32, 10 (2016), 1189-1202.

[16] J. H. Zheng, Value distribution of meromorphic functions, Tsinghua University Press, Beijing; Springer, Heidelberg, 2010. 